\title{
Bounded Verification of Ruby on Rails Data Models
}

\author{
Jaideep Nijjar and Tevfik Bultan \\ University of California, Santa Barbara \\ \{jaideepnijjar, bultan\}@cs.ucsb.edu
}

\begin{abstract}
The use of scripting languages to build web applications has increased programmer productivity, but at the cost of degrading dependability. In this paper we focus on a class of bugs that appear in web applications that are built based on the Model-View-Controller architecture. Our goal is to automatically discover data model errors in Ruby on Rails applications. To this end, we created an automatic translator that converts data model expressions in Ruby on Rails applications to formal specifications. In particular, our translator takes Active Records specifications (which are used to specify data models in Ruby on Rails applications) as input and generates a data model in Alloy language as output. We then use bounded verification techniques implemented in the Alloy Analyzer to look for errors in these formal data model specifications. We applied our approach to two open source web applications to demonstrate its feasibility.
\end{abstract}

\section{Categories and Subject Descriptors}

D.2.4 [Software Engineering]: Software/Program Verification-Formal methods; D.2.11 [Software Engineering]: Software Architectures-Data abstraction

\section{General Terms}

Verification

\section{Keywords}

automated verification, bounded verification, MVC frameworks, data model, web application modeling and analysis

\section{INTRODUCTION}

It has become common practice to write web applications using scripting languages, such as Ruby, because of their quick turnaround times in producing working applications. However, because of their dynamic nature, it is easy to introduce hard-to-find bugs to the applications written using these scripting languages. Current web software development processes rely on manual testing for eliminating bugs.

Permission to make digital or hard copies of all or part of this work for personal or classroom use is granted without fee provided that copies are not made or distributed for profit or commercial advantage and that copies bear this notice and the full citation on the first page. To copy otherwise, to republish, to post on servers or to redistribute to lists, requires prior specific permission and/or a fee.

ISSTA'11, July 17 - 21, 2011, Toronto, ON, Canada

Copyright 2011 ACM 978-1-4503-0562-4/11/07... \$10.00.
Although testing is necessary for improving the dependability of software systems in general, it is not possible to cover the state space of a web application using testing. Hence, undetected bugs find their way into deployed software systems resulting in unreliable behavior at best, and critical safety and security flaws at worst.

In this paper we present a bounded verification approach for data models of web applications that are written using the Ruby on Rails framework (Rails for short). Rails is a web development framework for the Ruby language, organized around the Model-View-Controller (MVC) architecture [4]. The MVC architecture facilitates the separation of the data model (Model) from the user interface logic (View) and the control flow logic (Controller). Due to the modularity and separation of concerns principles imposed by the MVC architecture, the data model specifications in Rails applications can be separated from the other application logic. This makes it feasible to perform verification on the data model of an application in isolation.

Our verification approach works as follows: We first automatically translate the Rails data models to formal specifications. Then, we write properties about the data model that we expect to hold. Next, we use bounded verification techniques to check if these properties hold on all the instances of the given data model within a given bound. We implemented this approach by writing a translator that translates the Rails data model specifications to Alloy specifications [16]. (Our translator targets Rails version 2 and the Alloy Analyzer version 4). We add the properties about the data model to this automatically generated Alloy specification. We then use the Alloy Analyzer to check these properties. The Alloy Analyzer converts bounded verification queries to Boolean SAT problems and uses a SAT-solver to determine the result.

To evaluate the effectiveness and usability of our approach, we applied it to two open-source Rails applications, TRACKS and FatFreeCRM. The model files of these two applications were fed into our tool to generate formal data model specifications. Using the Alloy Analyzer we found that some properties which could be enforced in the data model were not being enforced in these applications.

The rest of the paper is organized as follows: Section 2 describes the Rails data models. Section 3 formalizes the data model verification problem. Section 4 describes how 


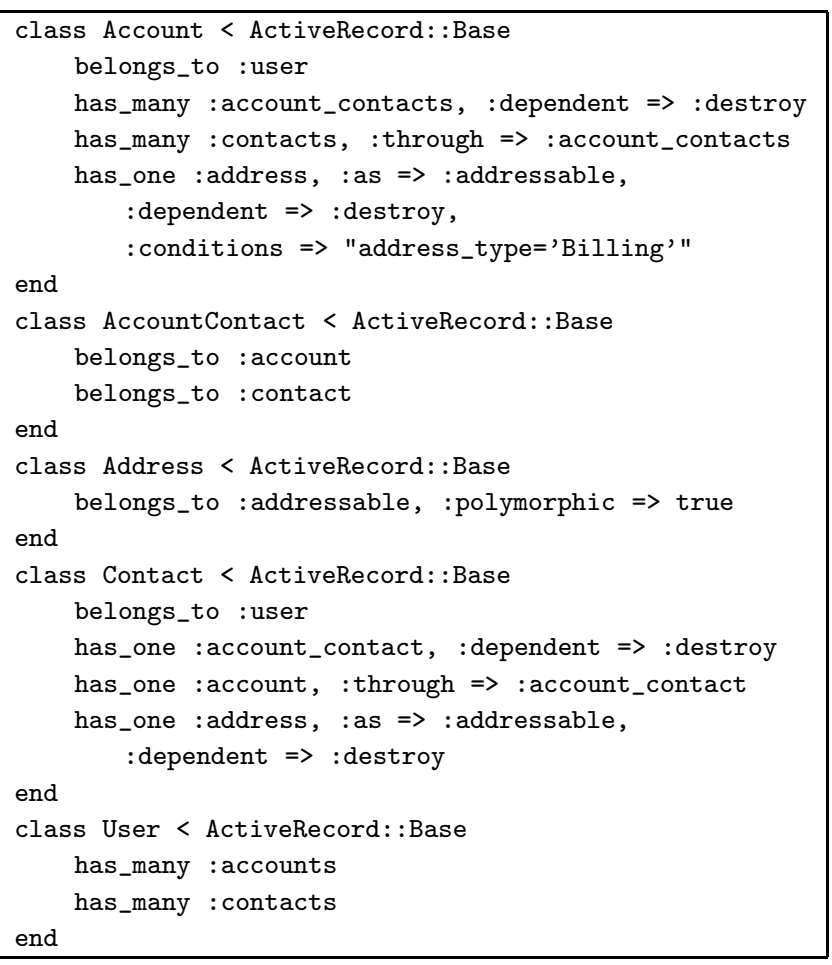

Figure 1: Simplified data model for FatFreeCRM

we translate Rails data models to the Alloy language. Section 5 describes our experiments. Section 6 discusses related work, and Section 7 presents our conclusions.

\section{RAILS DATA MODELS}

In this section, we describe how data models are expressed in Rails applications. The object-relational mapping Rails uses is called Active Records. Active Records handle all the details of connecting to the underlying database, mapping objects to tables, and data manipulation. Active Records are also used to manage relationships between tables.

We use the running example shown in Figure 1 to describe Rails' data-modeling features. This is a simplified version of the data model from an open source Customer Relations Management software called FatFreeCRM [12]. Customers are typically companies, and for each company an Account is created by a User. Users also create Contacts. Contacts are people in the companies who serve as the main person of contact for that company. Finally, each Contact and Account have an Address. (There is also an AccountContact class which is used to create a many-to-many relationship between Account and Contact, which we explain below.)

\subsection{Three Basic Relationships in Data Models}

Active Records support three basic types of relationships: 1) one-to-one: An Object $\mathrm{A}$ is associated with zero or one $\mathrm{Ob}-$ ject B's. So, more accurately, this is a one-to-zero-or-one relationship. 2) one-to-many: An Object A is associated with an arbitrary (zero or more) number of Object B's. 3) manyto-many: An arbitrary (zero or more) number of Object A's are associated with an arbitrary (zero or more) number of Object B's. These relationships are expressed by adding a pair of declarations (from the set of four declarations: has_one, has_many, belongs_to, and has_and_belongs_to_many) in the corresponding Rails models of the related objects:
1. one-to-one: Declaring a one-to-one relationship between Contact and AccountContact objects:

\begin{tabular}{l}
\hline class Contact < ActiveRecord: :Base \\
has_one : account_contact \\
end \\
\hline class AccountContact < ActiveRecord: :Base \\
belongs_to : contact \\
end
\end{tabular}

2. one-to-many: Declaring a one-to-many relationship between User and Account objects:

\begin{tabular}{l} 
class User < ActiveRecord: : Base \\
has_many : accounts \\
end \\
\hline class Account < ActiveRecord: : Base \\
belongs_to : user \\
end
\end{tabular}

3. many-to-many: Declaring a many-to-many relationship between Account and Contact objects ${ }^{1}$ :

\begin{tabular}{l}
\hline class Account < ActiveRecord: :Base \\
has_and_belongs_to_many : contacts \\
end \\
\hline class Contact < ActiveRecord: :Base \\
has_and_belongs_to_many :accounts \\
end
\end{tabular}

Note that in order to express the inheritance relation in Rails the notation ChildClass < ParentClass is used. Most objects will inherit from the ActiveRecord: : Base class, as seen in the examples above. This is so that the data objects inherit all the database-connection functionality that is located in the ActiveRecord class.

\subsection{Extending the Data Relationships}

Rails provides a set of options that can be used to extend the three basic relationships we presented above. Below we discuss the four options that affect relationships between data objects.

The : through Option. The first option we want to discuss is the :through option for the has_many and has_one declarations. Let us assume that we are using the :through option with the has_many declaration. The :through option is used when ObjectA has a one-to-many relation with ObjectB, ObjectC also has a one-to-many relation with ObjectB, and the Rails programmer would like direct access from ObjectA to ObjectC. For instance, let us say a data model is set up such that a Book has many Authors, and a Book also has many Editions. To get all the different editions of books an author has worked on, the programmer would have to write code to obtain the set of Books an Author has worked on, and then get the set of Editions of those Books. However, by using the : through option, the programmer can declare that Authors have many Editions : through Books. This will allow the programmer to directly access the set of book Editions from an Author object. Another use of the :through option is for setting up a many-to-many relation using a join model as opposed to a join table using the has_and_belongs_to_many declaration.

\footnotetext{
${ }^{1}$ Although FatFreeCRM does not have a direct manyto-many relationship between Accounts and Contacts, we present one here for illustrative purposes.
} 
We see this following use of the :through option between Accounts and Contacts in FatFreeCRM.

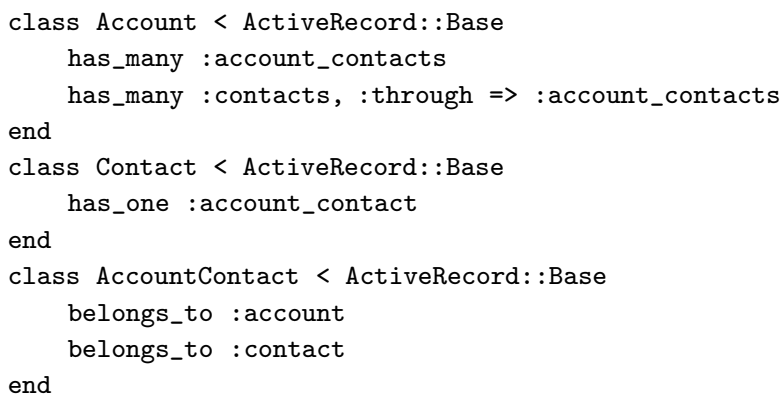

The : conditions Option. The second option that can be used to extend relationships is the : conditions option, which can be set on all of the four declarations (has_one, has_many, belongs_to, and has_and_belongs_to_many). As an example of its use, consider the following:

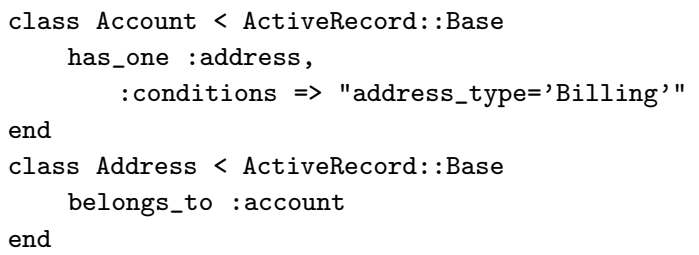

The : conditions option limits the relationship to those objects that meet a certain criteria. In this example, Account objects are only related to an Address object if the address_type field is Billing. The condition statement needs to be in the form of the WHERE clause of a SQL query.

The :polymorphic Option. Rails supports declaration of polymorphic associations. This is similar to the idea of interfaces in object oriented design, where we have dissimilar things that have common characteristics which are embodied in the interface they implement. In Rails, polymorphic associations are declared by setting the :polymorphic option on the belongs_to declaration. We see this setup in FatFreeCRM between Address, Accounts and Contacts. Although accounts and contacts are not similar enough to have a sub-class relationship, both have an address. By using the :polymorphic option in the Address class we set up an interface that allows any and all classes that have an address to create an association with the Address class. Any classes created in the future can also take part in this relationship, all without having to make any changes to the Address class. The models for the Account, Contact and Address classes are given below.

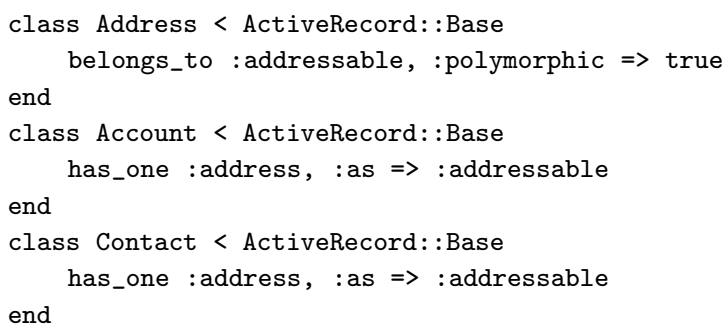

The polymorphic relationship is expressed in Account and Contact using the has_one declaration with an :as option. (The :as option can also be specified on a has_many declaration.)

The : dependent Option. The final Rails construct we want to discuss adds some dynamism to the data model; it allows modeling of object deletion at the data model level. The Rails construct for this is the :dependent option, which can be set for all the relation declarations except :has_and_belongs_to_many. Normally, when an object is deleted its related objects are not deleted. However, by setting the :dependent option to :destroy or :delete (:delete_all for has_many), deleting this object will also delete the associated object(s). Although there are several differences between :destroy and :delete, the one that is important for our purposes is that :delete will directly delete the associated object(s) from the database without looking at its dependences, whereas : destroy first checks whether the associated object(s) itself has associations with the :dependent option set. For an example of the use of the :dependent option, consider the following:

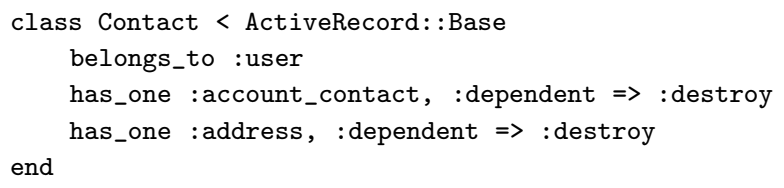

The Contact class has two relations with the :dependent option set. Thus, when a Contact object is deleted, the objects in these two relations, account_contact and address, will also be deleted. Further, since the :dependent option is set to : destroy for both these relations, any relations with the :dependent option set in the AccountContact and Address classes will also have their objects deleted.

\subsection{Data Model Properties}

The three basic relations and the four options that we have discussed above form the essence of the Rails data models. Note that using these constructs a developer can specify complex relationships among objects of a Rails application. Since a typical application would contain dozens or may be hundreds of object classes with many relationships among them, it is possible to have errors and omissions in the specification of the data model that may result in unexpected behaviors and bugs. Our goal in this paper is to develop an automated verification tool that can automatically analyze a Rails data model and identify errors. In order to look for errors though, we still need a specification of the properties that the developer expects to hold in the data model.

For example, for the FatFreeCRM running example, one property that we might want to check is the following: "Is it possible to have an account without any contacts?" The developer wants this behavior to be possible and may want to check that the data model is not over-constrained to prevent this behavior. Note that for this type of property finding an instance of the data model that satisfies the property would serve as proof of the property. However if we explore a set of instances and cannot find an instance that satisfies this property, we cannot definitely claim that the property fails 
since there might be an instance that satisfies the property that we have not explored.

As another example, consider the following property: "When an account is deleted, there will not be any contacts left without an account." I.e., all the contacts that are related to an account must be deleted with that account. The developer may want to make sure that the data model is not under-constrained such that a violation of this property is possible. For this type of property, if we find two consecutive instances of the data model such that one leads to the other by an account deletion and the second one violates the stated property, then we can be sure that the property is violated and there is an error in the data model. However, if we cannot find a violation of this property in a set of instances that we analyze, then that does not constitute a proof of the property.

\section{FORMALIZING DATA MODELS}

Based on the Active Record files in a Rails application, we can construct a formal data model representing the objects and their relationships in that application. Note that this data model is mainly a static model. In our analysis we do not model operations that update the objects and their relationships (and corresponding database records based on the object-relational mapping) except the delete propagation that is declared using the :dependent option. By focusing on the static data model specified in the Active Record files we can extract the set of constraints that must hold for any instance of the data model. In the rest of this section we present a formal data model and formalize the data model verification problem.

We define a data model as a tuple $M=\langle S, C, D\rangle$ where $S$ is the data model schema, identifying the sets and relations of the data model, $C$ is a set of relational constraints and $D$ is a set of dependency constraints. The schema $S$ only identifies the names of the object classes, the names of the relations and the domains and ranges of the relations in the data model. For example, the schema for the example shown in Figure 1 will identify the following set of object classes \{Account, AccountContact, Address, Contact, User $\}$ and the relations among these object classes \{accountaccount_contacts, account-contacts, account-address, contact-account_contact, contact-address, user-accounts, user-contacts $\}$ where each relation has an identified domain and range (we named the relations above so that the prefix identifies the domain and the suffix identifies the range).

The relational constraints in $C$ express all the constraints on the relations such as the ones related to cardinality (oneto-one, one-to-many, and many-to-many), the ones related to transitive relations (:through option), the ones related to conditional behavior (:conditions option), and the ones related to polymorphic behavior (:polymorphic option). For example, let us assume that $o_{A}$ and $o_{A C}$ are the set of objects for the Account and AccountContact classes and $r_{A-A C}$ is an instance of the relation account-account_contact. Since this is a one-to-one (i.e., one-to-zero-or-one) relation according to the declarations in Figure 1, then we would have the following relational constraint $C$ :

$$
\begin{aligned}
& \forall a c \in o_{A C}, \exists(a, a c) \in r_{A-A C} \\
\wedge & \forall a c \in o_{A C}\left((a, a c) \in r_{A-A C} \wedge\left(a^{\prime}, a c\right) \in r_{A-A C}\right) \Rightarrow a=a^{\prime} \\
\wedge & \forall a \in o_{A}\left((a, a c) \in r_{A-A C} \wedge\left(a, a c^{\prime}\right) \in r_{A-A C}\right) \Rightarrow a c=a c^{\prime}
\end{aligned}
$$

Note that this constraint states that each Account object must be associated with zero or one AccountContact object based on this relation, and each AccountContact object must be associated with exactly one Account object. If the relation $r_{A-A C}$ satisfies the above constraint, then we would state that $r_{A-A C} \models C$.

The dependency constraints in $D$ express conditions on two consecutive instances of a relation such that deletion of an object from one of them leads to the other instance by deletion of possibly more objects (based on the :dependent option). So, in order to determine if a dependency constraint holds, we need two instances of the same relation, say $r$ and $r^{\prime}$, one denoting the instance before the deletion, and one denoting the instance after the deletion, respectively. Then, if the pair of relations $\left(r, r^{\prime}\right)$ satisfy the dependency constraint, we write $\left(r, r^{\prime}\right) \models D$.

A data model instance is a tuple $I=\langle O, R\rangle$ where $O=\left\{o_{1}\right.$, $\left.o_{2}, \ldots o_{n_{O}}\right\}$ is a set of object classes and $R=\left\{r_{1}, r_{2}, \ldots r_{n_{R}}\right\}$ is a set of object relations and for each $r_{i} \in R$ there exists $o_{j}, o_{k} \in O$ such that $r_{i} \subseteq o_{j} \times o_{k}$.

Given a data model instance $I=\langle O, R\rangle$, we write $R \models C$ to denote that the relations in $R$ satisfy the constraints in $C$. Similarly, given two instances $I=\langle O, R\rangle$ and $I^{\prime}=\left\langle O^{\prime}, R^{\prime}\right\rangle$ we write $\left(R, R^{\prime}\right) \models D$ to denote that the relations in $R$ and $R^{\prime}$ satisfy the constraints in $D$.

A data model instance $I=\langle O, R\rangle$ is an instance of the data model $M=\langle S, C, D\rangle$, denoted by $I \models M$, if and only if 1 ) the sets in $O$ and the relations in $R$ follow the schema $S$, and 2) $R \models C$.

Given a pair of data model instances $I=\langle O, R\rangle$ and $I^{\prime}=$ $\left\langle O^{\prime}, R^{\prime}\right\rangle,\left(I, I^{\prime}\right)$ is a behavior of the data model $M=\langle S, C, D\rangle$, denoted by $\left(I, I^{\prime}\right) \models M$ if and only if 1$) O$ and $R$ and $O^{\prime}$ and $R^{\prime}$ follow the schema $\left.S, 2\right) R \models C$ and $R^{\prime} \models C$, and 3) $\left(R, R^{\prime}\right) \models D$.

Data Model Properties. Given a data model $M=\langle S, C, D\rangle$, we will define four types of properties: 1) state assertions (denoted by $A_{S}$ ): these are properties that we expect to hold for each instance of the data model; 2) behavior assertions (denoted by $A_{B}$ ): these are properties that we expect to hold for each pair of instances that form a behavior of the data model; 3) state predicates (denoted by $P_{S}$ ): these are predicates we expect to hold in some instance of the data model; and, finally, 4) behavior predicates (denoted by $P_{B}$ ): these are predicates we expect to hold in some pair of instances that form a behavior of the data model. We will denote that a data model satisfies an assertion or a predicate as $M \models A$ or $M \models P$, respectively. Then, we have the following formal 
definitions for these four types of properties:

$$
\begin{array}{rll}
M \models A_{S} \quad \Leftrightarrow \quad \forall I=\langle O, R\rangle, I \models M \Rightarrow R \models A_{S} \\
M \models A_{B} \Leftrightarrow \quad \forall\left(I=\langle O, R\rangle, I^{\prime}=\left\langle O^{\prime}, R^{\prime}\right\rangle\right), \\
& \left(I, I^{\prime}\right) \models M \Rightarrow\left(R, R^{\prime}\right) \models A_{B} \\
M \models P_{S} \quad \Leftrightarrow \quad \exists I=\langle O, R\rangle, I \models M \wedge R \models P_{S} \\
M \models P_{B} \Leftrightarrow \quad \exists\left(I=\langle O, R\rangle, I^{\prime}=\left\langle O^{\prime}, R^{\prime}\right\rangle\right), \\
& \left(I, I^{\prime}\right) \models M \wedge\left(R, R^{\prime}\right) \models P_{B}
\end{array}
$$

Bounded Verification of Data Models. The data model verification problem is, given one of these types of properties, determining if the data model satisfies the property. Since the number of objects in a data model is not bounded, we cannot enumerate all the instances in a data model. One possible approach is to use bounded verification where we check the property for instances within a certain bound. This is the approach we take in this paper. The main idea is to bound the set of data model instances to a finite set, say $\mathcal{I}_{k}$ where $I=\langle O, R\rangle \in \mathcal{I}_{k}$ if and only if for all $o \in O$ $|o| \leq k$. Then given a state assertion $A_{S}$, we can check the following condition for example:

$$
\exists I=\langle O, R\rangle, I \in \mathcal{I}_{k} \wedge I \models M \wedge R \not \models A_{S}
$$

Note that if this condition holds then we can conclude that the assertion $A_{S}$ fails for the data model $M$, i.e., $M \not$ $A_{S}$. However, if the above condition does not hold, then we only know that the assertion $A_{S}$ holds for the data model instances in $\mathcal{I}_{k}$.

Similarly, given a predicate $P_{S}$, and a bounded set of instances $\mathcal{I}_{k}$, we can check the condition:

$$
\exists I=\langle O, R\rangle, I \in \mathcal{I}_{k} \wedge I \models M \wedge R \models P_{S}
$$

and if this condition holds we can conclude $M \models P_{S}$. If the above condition fails on the other hand, we can only conclude that the predicate $P_{S}$ does not hold for the data model instances in $\mathcal{I}_{k}$. Bounded verification of behavior assertions and behavior predicates can also be done similarly on bounded data model instances.

An enumerative (i.e., explicit state) search technique is not likely to be efficient for bounded verification since even for a bounded domain the set of data model instances can be exponential in the number of sets in the data model. One bounded verification approach that has been quite successful is SAT-based bounded verification. The main idea is to translate the verification query to a Boolean SAT instance and then use a SAT solver to search the state space. Alloy Analyzer [16] is a SAT-based bounded verification tool for analyzing object-oriented data models. Alloy language allows specification of objects and relations and it allows specification of constraints on relations using first-order logic. Alloy analyzer supports bounded verification of assertions and simulation of predicates which correspond to the assertion and predicate checks we described above. In order to do bounded verification of Rails data models, we implemented an automated translator that translates Active Record specifications to Alloy specifications. After this automated translation, we use the Alloy Analyzer for bounded verification of data model properties. Below we describe how we translate the Active Record specifications to the Alloy language.

\section{TRANSLATION TO ALLOY}

We implemented a translator that translates data models in Rails applications to Alloy. The first step of the translation is parsing the Rails model files (i.e., Active Record files). We do this using a parser written in and for Ruby source code called ParseTree [18]. ParseTree extracts the parse tree for an entire Ruby class and returns it as an s-expression. S-expressions are generated for each model file that contains a class that inherits from ActiveRecord. We then create an s-expression processor (which inherits from SexpProcessor, the basic s-expression traversal class provided with ParseTree) to traverse the generated s-expressions and translate them to a single Alloy specification file.

The first step of the Active Record to Alloy translation is to map each Active Record class to a sig in Alloy, which simply defines a set of objects in Alloy. The inheritance relationships in Rails, such as class Child < Parent, are translated to Alloy using the extends keyword, as in class Child extends Parent.

The Three Basic Relationships. When expressing a binary relationship in Alloy, one can give it a multiplicity of one, lone, some, or set which correspond to one, zero or one, one or more, and zero or more, respectively. Thus, the mapping of the Rails relationships to Alloy is as follows:

\begin{tabular}{|l|l|}
\hline $\begin{array}{l}\text { class ObjectA } \\
\text { has_one :objectB } \\
\text { end }\end{array}$ & $\begin{array}{l}\text { sig ObjectA \{ } \\
\text { objectB: lone ObjectB }\end{array}$ \\
\hline $\begin{array}{l}\text { class ObjectA } \\
\text { has_many :objectBs } \\
\text { end }\end{array}$ & $\begin{array}{l}\text { sig ObjectA \{ } \\
\text { objectBs: set ObjectB }\end{array}$ \\
\hline $\begin{array}{l}\text { class ObjectA } \\
\text { belongs_to :objectB } \\
\text { end }\end{array}$ & $\begin{array}{l}\text { sig ObjectA \{ } \\
\text { objectB: one ObjectB }\end{array}$ \\
\hline $\begin{array}{l}\text { class ObjectA } \\
\text { has_and_belongs_to_many } \\
\text { objectBs }\end{array}$ & $\begin{array}{l}\text { sig ObjectA \{ } \\
\text { objectBs: set ObjectB }\end{array}$ \\
end & \} \\
\hline
\end{tabular}

Furthermore, one has to add a fact block that connects each pair of declarations. For the one-to-many relationship this would look as follows:

fact $\{$ Object $\mathrm{A}<:$ objectBs $=\sim($ Object $\mathrm{B}<: \quad$ object $A)\}$ where $<$ : is the domain restriction operation such that $\mathrm{s}<$ : $r$ contains the tuples in relation $r$ that start with an element in $\mathbf{s}$, and the operator $\sim$ is the relational inverse operation where $\sim r$ is the inverse of the relation $r$ [16].

The :through Option. To translate the :through option, we follow the mapping from the table in the previous paragraph. However, instead of a separate global fact block, we add a local fact block immediately following the signature of the object containing the :through declaration. This is because all the fields referred to in the fact refer to the those inside that single signature. So, for the following Rails models:

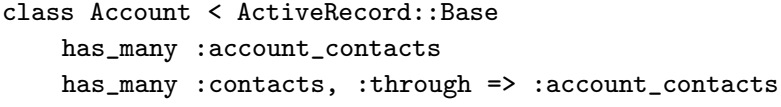




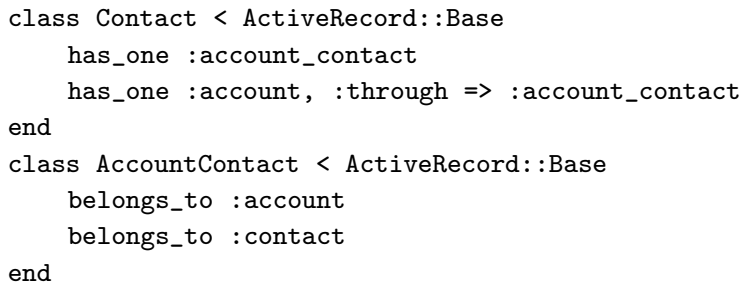

the Alloy translation looks as follows:

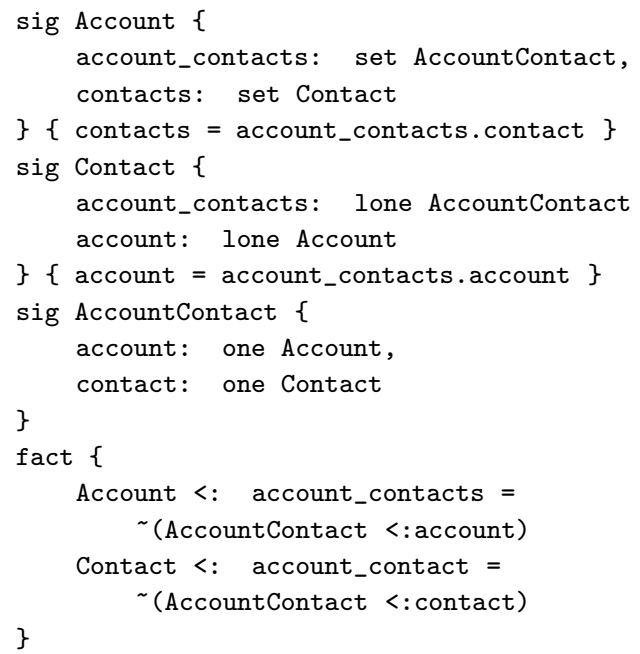

The :conditions Option. The :conditions option means that objects from one class only associate with a subset of objects from another class rather than with the entire set. Thus, to translate the :conditions option we create a subset of objects in Alloy which the object with the condition statement can map to. Therefore if we had the following Rails models:

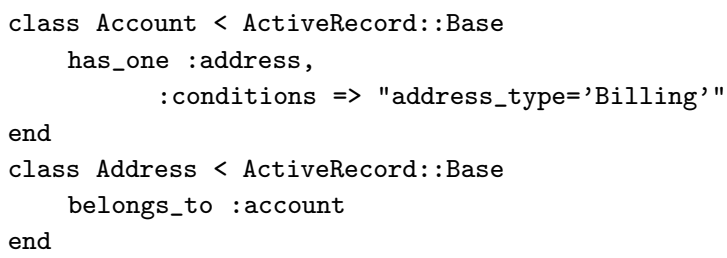

we translate it to Alloy by abstracting the set of addresses for which the condition address_type='Billing' holds, to the set Billing_Address as follows:

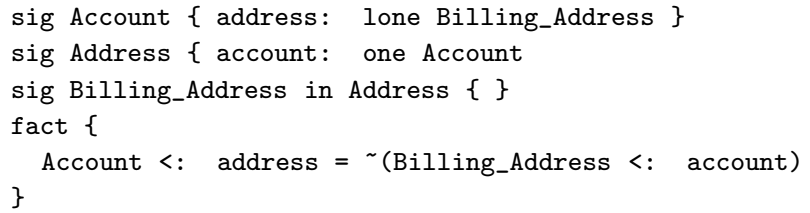

The in keyword in Alloy creates a subset; it is used above to create the Billing_Address signature. Since we are not modeling the data fields of the Rails classes, we create this arbitrary subset of Address without specifying exactly which elements of Address belong in the subset (i.e. the ones which have 'Billing' as the address_type). The address element in Account can now map to just this subset of Address. The global fact block establishes this mapping by confirming the address and account fields of the two signatures refer to the same set of objects.

The :polymorphic Option. In polymorphic relations, there is a base class that can be related to one of many target classes. Moreover, this relationship is expressed via a single field in the base class. So, to translate the polymorphic relation, we need to enclose the target classes inside a single supertype which the relation in the base class can refer to. However the translation for polymorphic relations is not straightforward since a target class can have polymorphic relations with multiple classes. Modeling these kinds of scenarios requires multiple inheritance.

To understand how to simulate multiple inheritance in Alloy, let us assume that the class Contact needs to inherit from both Addressable and Subject. In order to simulate multiple inheritance, all Active Record classes are made a subset of some other superclass, say ActiveRecord. We will use the extends keyword in Alloy to ensure the subsets are disjoint. Then statements are added to the global fact block which will say Contact is a subset of both Addressable and Subject; but this time we will use the in keyword to declare the subset, which will allow overlapping (as opposed to the extends keyword, which forces the subsets to be disjoint).

Let us take a look at a concrete example from FatFreeCRM. Below are a set of Rails models with a polymorphic association. Address has an addressable association that both Account and Contact refer to:

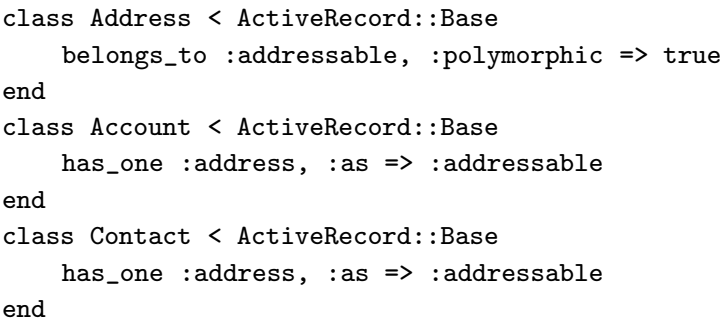

The first step in translating these models is to create a common base class that all classes extend, as follows:

abstract sig ActiveRecord \{\}

The abstract keyword tells Alloy that this signature has no elements except those belonging to its extensions. All signatures will either inherit from this class or the parent class if one is specified in the corresponding Rails model.

The next step is to create a supertype for the target classes to be enclosed in. The supertype will be called Addressable and it will contain the has_one relation, translated as described earlier:

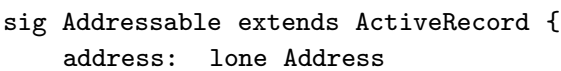

Next, the relationship between Addressable and the target classes (Contact and Account) will be established via facts. Specifically, we will state that the target classes are subsets of Addressable, using the in keyword. 
Further, Alloy does not allow subsets to be abstract if the superset is abstract, like we have made ActiveRecord. Thus we will also have to specify as facts that there are no elements in Addressable except those belonging to the target classes. Finally, since our design requires all signatures to extend ActiveRecord, we also have to add facts to state that Addressable is disjoint from all other non-target classes in ActiveRecord. The final Alloy translation is given below.

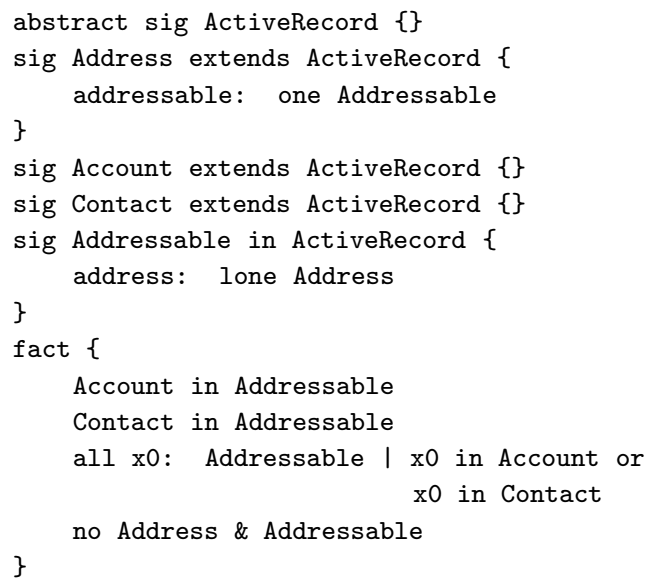

\subsection{Translating the Dependency Constraints}

Finally we have the :dependent option, which specifies what behavior to take on deletion of an object with regards to its associated objects. To incorporate this dynamism, the model must allow analysis of how sets of objects and their relations change from one state to the next. Thus we need a slightly different translation algorithm from the one we have been presenting so far.

In order to handle the :dependent option, we will be creating invokable constraints, or predicates in Alloy, which will model the deletion of an object. We will also need Alloy signatures to represent the state of a data model instance, i.e. the set of all objects and their relations. In particular, we will have a PreState signature to represent the state of objects before the deletion operation, and a PostState signature to represent the state after the deletion. We can then use these signatures to check whether some invariant holds after an object is deleted.

Basic Translation. We will use snippets of the running example to explain each piece of this new translation algorithm. Let us begin with the following portion of the Rails data model for FatFreeCRM:

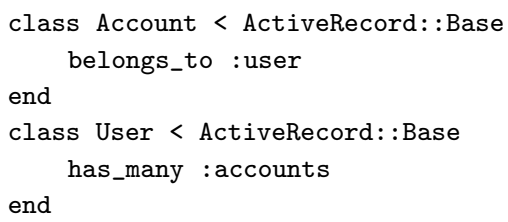

As before, the Alloy specification for this model will contain a signature for each class. It will also contain a PreState and a PostState signature, as just discussed. Since the PreState and PostState signatures represent the whole data model instance, they will need references to all object types and relations. Thus we obtain the following Alloy specification:

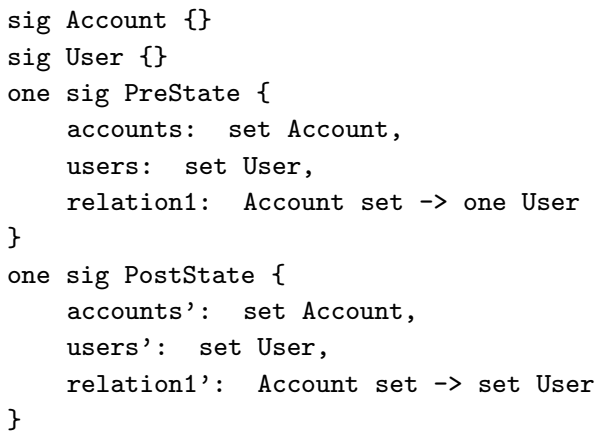

The PreState sig contains fields accounts and users to hold objects of each type in the system. Next, it contains a field relation1 to hold the related Account and User objects. The product operator, $->$, produces a mapping between Accounts and Users. The multiplicity keyword set tells Alloy that relation1 maps each User object to zero or more Account objects, and the keyword one tells Alloy that every Account object is mapped to exactly one User object. Note that in the translation of relations, the multiplicity keywords are the same as the ones used in the earlier translation (e.g. belongs_to : user produces one User and has_many :accounts produces Account set). Also note the one preceding sig PreState. This tells Alloy that there will be exactly one instance of PreState in any data model instance.

The definition of PostState is exactly the same. The only difference is that its relations always map a set of objects to another set of objects. The reason to not specify the relation cardinalities here as well is because when the cardinality is one, it forces the mapping to be total. However once an object has been deleted, we need to remove it from the relation, causing the need for a partial mapping in the PostState. Since the relations in PostState will be defined in the delete predicates using the PreState relations, the cardinalities among the remaining (live) objects will be preserved.

Let us now turn to the definition of the delete predicates. As an example, let us generate the predicate that deletes an Account. To start, we define the deleteAccount predicate to accept a PreState object, a PostState object and an Account object as parameters. The body of the predicate begins by stating that $\mathrm{s}$, the PreState object, contains all existing objects:

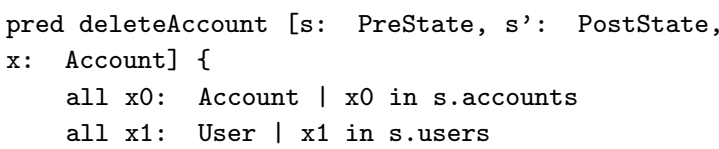

Finally, we describe the data model instance after the deletion:

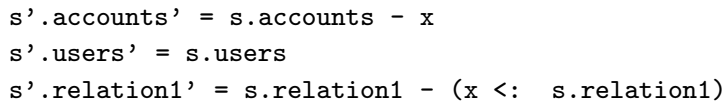

Here we have deleted $\mathrm{x}$, the Account object, by removing it from the set of Account objects in PostState. We have also updated the relation by setting the PostState relation to be the PreState relation minus all the tuples whose domain is 
$\mathrm{x}$ (using the scoping operator $<$ : described earlier). This removes all of x's relations from relation1'.

It is important to note here that the relation is only updated if it is a :belongs_to or :has_and_belongs_to_many relationship in the Rails model. (So in the delete predicate for User which contains the has_many declaration, relation1 would remain unchanged: s'.relation1' = s.relation1.) This is due to the way the relationships are implemented in Rails. In the database, the foreign key is stored with the object that has the : belongs_to relationship (for the one-to-one and one-tomany relations) or in a join table for the :has_and_belongs_to_many relationships. Thus, an object's has_one andhas_many relations are not affected when an object is deleted. Note that deleting an object on the has_one or has_many side may cause a dangling reference if the :dependent option is not set; our model can be used to check for such cases (as we did in our case studies).

The :through Option. Next, let's analyze the following partial Rails model to understand how to translate the : through option for the dynamic Alloy specification.

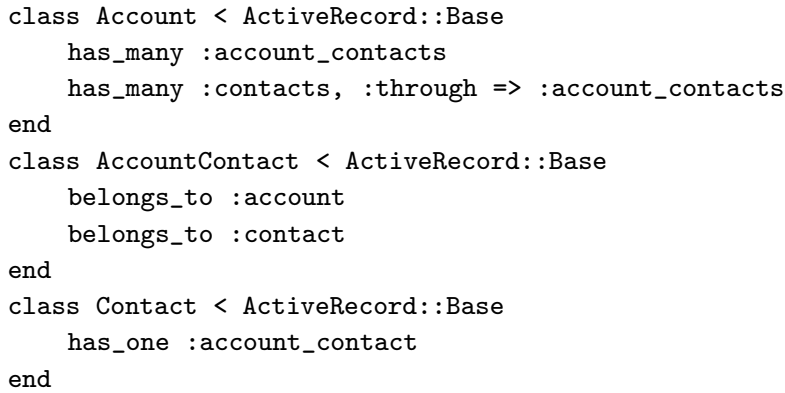

The basic setup for the Alloy specification is the same: a signature for each class, a PreState and PostState signature, each with a field for every set of objects and relations between them.

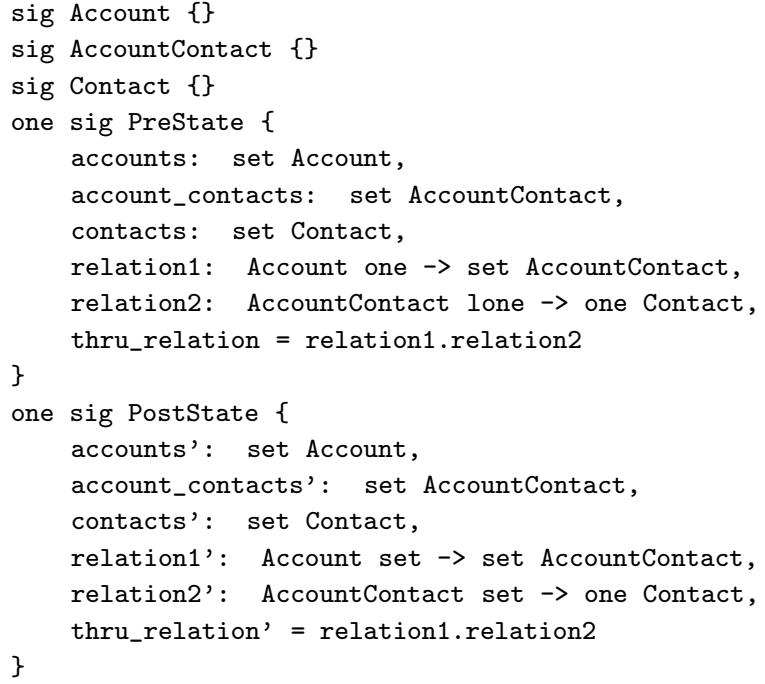

The new idea is the translation of the relation with the : through option set, the one between Account and Contact. We use the join operator, ., to define thru_relation to be the join of the other two relations.
The definition of the delete predicate (provided below) is also basically the same. Let's say we want to delete an AccountContact. We delete the object from PostState's set of account_contacts. We also delete it from any of the object's :belongs_to or :has_and_belongs_to-many relations. Incidentally, both of its relations are :belongs_to; thus, tuples containing $\mathrm{x}$ in both the relations relation1 and relation2 are removed. Note that thru_relation does not need to be updated explicitly; it will be updated automatically because it is defined using relation1 and relation2.

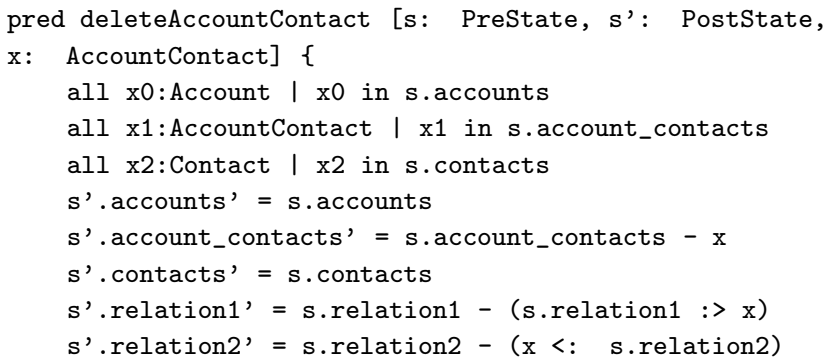

The :conditions, :polymorphic, and :dependent Options. The translation of the :conditions and the : polymorphic options remain the same as described in the previous translation, except that they contain the PreState and PostState sigs. A relation with the :dependent option is translated using the guidelines we discussed above; the only change is in the delete predicate. When updating the relations by removing those belonging to the deleted object, we also update relations of its associated object(s) based on the use of the : dependent option.

\section{EXPERIMENTATION}

We used our Active Records to Alloy translator tool and the Alloy Analyzer to analyze data models of two open source Ruby on Rails applications, TRACKS [21] and Fat Free CRM [12].

TRACKS is an application to manage things-to-do lists. It has 6062 lines of code, with 44 total classes and 13 data model classes. TRACKS allows users to organize to-do items by context or project. Notes can be added to to-do lists and projects. TRACKS is also multi-user. The Alloy specification that our translator produced for this application contains 444 lines of code.

Fat Free CRM is roughly twice as big as TRACKS, with 12069 lines of code, 54 classes and 20 data model classes. The Alloy translation has 1518 lines of code. Fat Free CRM aims to be a lightweight solution to customer relationship management (CRM). Fat Free CRM, offers the management of leads (a person who is a potential customer), accounts, opportunities and campaigns All of this is handled within a multi-user environment.

Property Classification. The model files of these two applications were fed into our automatic translator, which generates an Alloy specification. To this, we added properties about the data model and relations between objects. We had four basic classes of properties, which we describe below: 
I. Relationship Cardinality: These properties check the cardinality of a relationship. For instance, the application specification may require there be a one-to-one relationship between two objects. This can be the cause of bug if the Rails programmer is not aware that Ruby on Rails' one-to-one relationship is actually a one-to-zero-or-one relationship.

II. Transitive Relations: This class of properties check whether the set of objects in a direct relationship between two objects is the same as the set of objects obtained from an indirect, or transitive, relationship. This class of properties can fail due to incorrect usage of the :through option. In TRACKS for instance, Notes belong to Users; Notes also belong to Projects, and Projects belong to a User. So one can check whether the User of a Note is the same as the Note's Project's User, for all Notes.

III. Deletion Does Not Create Orphans: We also verified properties that checked whether deleting an object caused a related object to be orphaned. This happens when semantically two objects should always be related, but the application allows one of them to be deleted.

IV. Deletion Does Not Cause Dangling References: Another class of properties we checked were whether deleting an object caused dangling references. This may happen due to forgotten : dependent declarations on the has_many or has_one side of a relationship.

V. Deletion Propagates to Associated Objects: Finally, we checked whether deleting an object also caused certain related objects to be deleted (or that the associated objects did not get deleted if they should not have). Again, this type of properties may fail due to incorrect usage of the :dependent option. One can also verify that an associated object still exists after the delete.

The complete list of properties verified for both applications is shown in Table 1. It includes a description of the property, whether it passed or failed during verification, and which of the above categories it belongs to. It also includes the type of property based on the types we defined in Section 3.

Property Classification. In the TRACKS Application, a total of ten properties were checked. Of the ten properties, five failed. Of these that failed we considered it a data modeling error if the property could have been enforced by the data model but was not.

The first property that failed is property T4. Inspection of the data models reveals that the relationship between a Todo and Projects is one-to-many; the has_many/belongs_to declaration pair is used. However, the application actually requires a zero-or-one-to-many relationship between Todo and Projects. Looking at the data models shows that to make up for Rails' limited expressive power for relations, the application programmer has enforced this property in the data model by adding code to return a NullProject. ${ }^{2}$ This code is not part of the static data model so our analysis does not model it. Since this property cannot be enforced

\footnotetext{
${ }^{2}$ An empty Project that represents the absence of an object.
} This is preferable over a null reference in some cases.

\begin{tabular}{|l|l|l|}
\hline Type & TRACKS Properties & \\
\hline $\mathrm{I}, A_{S}$ & T1 Every Todo has a Context & $\mathrm{P}$ \\
\hline $\mathrm{I}, P_{S}$ & T2 A Context may have no Todos & $\mathrm{P}$ \\
\hline $\mathrm{I}, A_{S}$ & T3 A Todo must have a Context & $\mathrm{P}$ \\
\hline $\mathrm{I}, P_{S}$ & T4 A Todo can have no Project & $\mathrm{F}$ \\
\hline $\mathrm{II}, A_{S}$ & T5 Note's User = Note's Project's User & $\mathrm{F}$ \\
\hline $\mathrm{I}, A_{S}$ & T6 Every User has a Preference & $\mathrm{F}$ \\
\hline $\mathrm{IV}, A_{B}$ & T7 No dangling Todos after User delete & $\mathrm{P}$ \\
\hline $\mathrm{III}, A_{B}$ & T8 No orphan User after Preference delete & $\mathrm{F}$ \\
\hline $\mathrm{IV}, A_{B}$ & T9 No dangling Todos after Context delete & $\mathrm{P}$ \\
\hline $\mathrm{IV}, A_{B}$ & T10 No dangling Todos after User delete & $\mathrm{F}$ \\
\hline \hline Type & FFCRM Properties & \\
\hline $\mathrm{I}, A_{S}$ & F1 An Opportunity must have a Campaign & $\mathrm{P}$ \\
\hline $\mathrm{I}, A_{S}$ & F2 A Task must have a User & $\mathrm{P}$ \\
\hline $\mathrm{I}, P_{S}$ & F3 An Account may have no Activities & $\mathrm{P}$ \\
\hline $\mathrm{I}, A_{S}$ & F4 At most one User per Lead & $\mathrm{P}$ \\
\hline $\mathrm{II}, A_{S}$ & $\begin{array}{l}\text { F5 A ContactOpportunity's Opportunity }= \\
\text { ContactOpportunity's Contact's Opportunity }\end{array}$ & $\mathrm{P}$ \\
\hline $\mathrm{I}, P_{S}$ & F6 A Contact may have no Tasks & $\mathrm{P}$ \\
\hline $\mathrm{I}, A_{S}$ & F7 A Contact cannot have two Accounts & $\mathrm{P}$ \\
\hline $\mathrm{II}, A_{S}$ & $\begin{array}{l}\text { F8 User's Opportunities } ~ \\
\text { User's Campaigns' Opportunities }\end{array}$ \\
\hline $\mathrm{II}, A_{S}$ & F9 Lead's User = Lead's Activities' User & $\mathrm{F}$ \\
\hline $\mathrm{IV}, A_{B}$ & F10 No dangling Contacts after Account delete & $\mathrm{P}$ \\
\hline $\mathrm{V}, A_{B}$ & $\begin{array}{l}\text { F11. Deleting an Account does not delete its } \\
\text { Contacts }\end{array}$ & $\mathrm{P}$ \\
\hline $\mathrm{V}, A_{B}$ & F12 Deleting an Account deletes its Todos & $\mathrm{P}$ \\
\hline $\mathrm{V}, A_{B}$ & F13 Deleting a Campaign deletes its Leads & $\mathrm{P}$ \\
\hline $\mathrm{V}, A_{B}$ & $\begin{array}{l}\text { F14 Deleting a Campaign deletes its } \\
\text { Opportunities }\end{array}$ & $\mathrm{P}$ \\
\hline $\mathrm{V}, A_{B}$ & F15 Deleting Campaign deletes its Leads' Tasks & $\mathrm{P}$ \\
\hline $\mathrm{IV}, A_{B}$ & F16 No dangling Contacts after Lead delete & $\mathrm{F}$ \\
\hline $\mathrm{V}, A_{B}$ & $\begin{array}{l}\text { F17 Deleting a Lead does not delete its } \\
\text { Contacts }\end{array}$ & $\mathrm{P}$ \\
\hline $\mathrm{IV}, A_{B}$ & F18 No dangling Users after Lead delete & $\mathrm{P}$ \\
\hline $\mathrm{V}, A_{B}$ & F19 Deleting a Lead does not delete its User & $\mathrm{P}$ \\
\hline $\mathrm{V}, A_{B}$ & $\begin{array}{l}\text { F20 Deleting a User means its associated } \\
\text { Activities are also deleted }\end{array}$ & $\mathrm{P}$ \\
\hline
\end{tabular}

Table 1: Verification Results (P:Pass, F: Fail)

in a Rails data model we do not consider this failure a datamodeling error.

The next property to fail is T5. In the TRACKS application, there is a relationship between Users and Projects, Users and Notes, and Notes and Projects. This property checks that the User a Note belongs to is the same User that the Note's Project is associated with. Analysis of the failure of this property shows that there is a flaw in the design of the data model. The user field is duplicated in the Project and Note classes. This was probably done for ease of access of the User from the Note class, as opposed to going through the Project class. Currently, this property is being upheld in the application by the code placed in the controller. However, in the future if the controller code is changed and the programmer is unaware of this invariant, a bug may be introduced into the application. This property could have been enforced in the data model using the :through option, hence we consider this failure a data-modeling error.

The third property that failed verification in TRACKS is property $\mathrm{T} 6$. In the data models, one observes that the relationship between User and Preference is set up using has_one and belongs_to. Recall that this is a one-to-zero-or- 
one relationship. However, in TRACKS, this relationship is actually one-to-one. Once again we observe the limitation of Rails' ability to express relationships of this kind. Although this constraint is being enforced in the controller, there are ways of enforcing the one-to-one relationship in the data models. For example, the User and Preferences tables can be merged or a construct in Rails called callbacks may be used. Consequently, this property failure also points out a data-modeling error in the application. Property T8 failed due to this same data modeling error. A User should always be related to a Preference, but allowing a Preference to be deleted breaks this property.

The final property that failed verification in TRACKS is property $\mathrm{T} 10$. In the data models, we see that there is a relationship where a User has_many Contexts, and a Context has_many Todos. Because the has_many relation in User has the :dependent option set to :delete_all, the associated Context objects will be deleted, but Rails will not go into Context and look at its relations. Thus, the User deletion does not get propagated into the relations of the Context object, including the Todos that this property is checking for. Hence, this property pinpoints another data-modeling error. However, the User object itself also is related to Todos, and since the application has controller logic that enforces this set of Todos is the same as the ones obtained by navigating through the Context relation, the application currently does not manifest this data modelling error.

For the Fat Free CRM application, twenty properties were verified. Of these, two did not pass the verification. The first is F8, and the situation for this property is the same as property T5 of TRACKS. Hence this property points to a data-modeling error.

The other property that fails for Fat Free CRM is property F16. Leads and Contacts are associated such that a Lead has_one Contact, with no :dependent option set on the association. Thus, when a Lead is deleted, the associated Contact still exists. A comment next to the relation declaration confirms that the application programmer wanted the Contact to remain alive. However, this Contact now has a null reference and thus breaks the model design; the use of the has_one/belongs_to declaration pair signifies a oneto-zero-or-one relationship, whereas it seems as if the application programmer wanted a zero-or-one-to-zero-or-one relationship. Further inspection of the code reveals that this relationship serves no purpose. In the application logic, some Leads may be upgraded to Contacts, which means (in the code) that a Contact object is created from a Lead object by copying many of the fields over. Hence, a Contact never refers back to the Lead that created it, nor the Lead to the Contact it created. Perhaps this relationship seemed necessary during the initial drafts of the data model design; however, it is now extraneous and only allows room for future bugs. Thus, the violation of this property uncovers a data-modeling error.

Performance. To measure performance, we recorded the amount of time it took for Alloy to run and check the properties on a bounded number of instances, as well as the number of variables and clauses generated in the boolean formula generated for the SAT-solver. The time, number of variables and number of clauses are averaged over the properties for each application. These performance measures are taken over an increasing bound, from at most 10 objects for each class to at most 35 objects for each class. This means that each property was checked by instantiating up to 10 instances of each object, up to 15 of each object, etc The results are summarized in Figure 2. We see that the number of variables and clauses, and verification time increase with increasing bound as expected. In the worst case, this increase could be exponential. However, the slowest verification time (for a bound of 35 objects) being only 22 seconds confirms that the bounded analysis we are performing is feasible for analyzing properties of real-world web applications.

\section{RELATED WORK}

Formal modeling and automated verification of web applications has been investigated before. There has been some work on analyzing navigation behavior in web applications, focusing on correct handling of the control flow given the unique characteristics of web applications, such as the use of a browser's "back" button combined with the stateless nature of the underlying HTTP protocol [17]. Prior work on formal modeling of web applications mainly focuses on state machine based formalisms to capture the navigation behavior. Modeling web applications as state machines was suggested a decade ago [20] and investigated further later on [14, $2,13]$. State machine based models have been used to automatically generate test sequences [23], perform some form of model checking [19] and for runtime enforcement [13]. In contrast to these previous efforts, we are focusing on analysis of the data model rather than the navigational aspects.

There has been some prior work on formal modeling of web applications using UML [7] and extending UML to capture complex web application behavior such as browsing and operations on navigation states [1]. WebML [5] is a modeling language developed specifically for modeling web applications. Formal specification of access control policies in conjunction with a data model using Alloy has also been studied, where an implementation is automatically synthesized from the formal specification [6]. These efforts focus on model driven development whereas our approach is a reverse engineering approach that extracts the model of an already-existing application and analyzes it to find errors.

The verification of traditional, non-MVC web applications, has also been investigated in recent years $[15,9,10,11]$. In this paper, by focusing on MVC style web applications we are able to exploit the modularity in the MVC architecture and extract formal data models from existing applications.

There has been some prior work on using Alloy for data model analysis. For example, mapping relational database schemas to Alloy has been studied before [8]. Also, translating ORA-SS specifications (a data modeling language for semi-structured data) to Alloy and using Alloy analyzer to find an instance of the input data model has been investigated [22]. However, unlike our work, the translation to Alloy is not automated in these earlier efforts. Finally, Alloy has also been used for discovering bugs in web applications related to browser and business logic interactions [3]. This is a different class of bugs than the data model related bugs we focus on in this paper. 

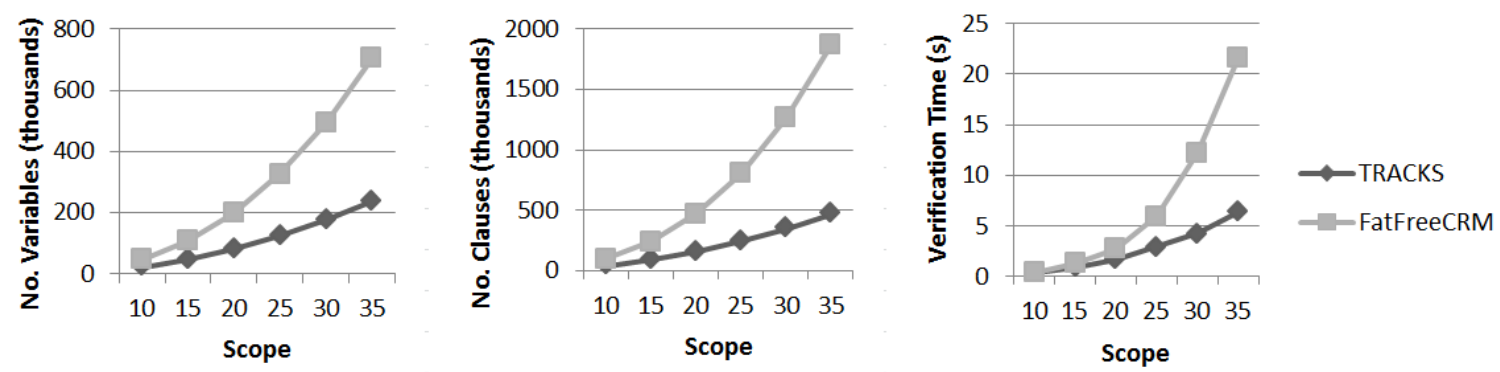

Figure 2: Verification Performance with Increasing Bound (Scope)

\section{CONCLUSIONS}

We presented techniques for bounded verification of Ruby on Rails data models. We showed that by exploiting the inherent modularity in MVC frameworks it is possible to extract a static data model from a Rails application. We formalized the bounded verification problem for Rails data models and realized a bounded verification framework by implementing an automated translator from Rails data models to Alloy language. The Alloy specification that our translator outputs can then be appended with properties about the data relationships and checked by the Alloy Analyzer. We applied this approach to two open source web applications and demonstrated that this type of bounded verification is feasible and can discover data modeling errors in real applications. As future work we plan to investigate fusing the data model investigated in this paper with a navigation model in order to analyze dynamic data model behavior.

\section{REFERENCES}

[1] L. Baresi, F. Garzotto, and P. Paolini. Extending UML for modeling web applications. In Proc. 34th Ann. Hawaii Int. Conf. Sys. Sci. (HICSS), 2001.

[2] M. Book and V. Gruhn. Modeling web-based dialog flows for automatic dialog control. In Proc. 19th Int. Conf. Automated Software Engineering (ASE), pages 100-109, 2004.

[3] B. Bordbar and K. Anastasakis. MDA and analysis of web applications. In Proc. VLDB Workshop on Trends in Enterprise Application Architecture, pages 44-55, 2005.

[4] F. Buschmann et al. Pattern-Oriented Software Architecture: A System of Patterns. John Wiley and Sons, 1996.

[5] S. Ceri, P. Fraternali, and A. Bongio. Web modeling language (WebML): a modeling language for designing web sites. Computer Networks, 33(1-6):137-157, 2000.

[6] F. Chang. Generation of Policy-rich Websites from Declarative Models. PhD thesis, MIT, 2009.

[7] J. Conallen. Modeling web application architectures with UML. Commun. ACM, 42(10):63-70, 1999.

[8] A. Cunha and H. Pacheco. Mapping between Alloy specifications and database implementations. In Proc. 7th Int. Conf. Engineering and Formal Methods (SEFM), pages 285-294, 2009.

[9] L. Desmet, P. Verbaeten, W. Joosen, and F. Piessens. Provable protection against web application vulnerabilities related to session data dependencies. IEEE Trans. Software Eng., 34(1):50-64, 2008.
[10] A. Deutsch, L. Sui, V. Vianu, and D. Zhou. A system for specification and verification of interactive, data-driven web applications. In Proc. ACM SIGMOD Int. Conf. on Management of Data, pages 772-774, 2006.

[11] F. M. Donini, M. Mongiello, M. Ruta, and R. Totaro. A model checking-based method for verifying web application design. Electr. Notes Theor. Comput. Sci., 151(2):19-32, 2006.

[12] Fat free crm. http://www.fatfreecrm.com/.

[13] S. Hallé, T. Ettema, C. Bunch, and T. Bultan. Eliminating navigation errors in web applications via model checking and runtime enforcement of navigation state machines. In Proc. 25th Int. Conf. Automated Software Engineering (ASE), pages 235-244, 2010.

[14] M. Han and C. Hofmeister. Relating navigation and request routing models in web applications. In 10th Int. Conf. on Model Driven Engineering Languages and Systems (MoDELS), pages 346-359, 2007.

[15] M. Haydar. Formal framework for automated analysis and verification of web-based applications. In Proc. 19th Int. Conf. Automated Software Engineering (ASE), pages 410-413, 2004.

[16] D. Jackson. Software Abstractions: Logic, Language, and Analysis. The MIT Press, Cambridge, Massachusetts, 2006.

[17] S. Krishnamurthi, R. B. Findler, P. Graunke, and M. Felleisen. Modeling Web Interactions and Errors, pages 255-275. Springer, 2006.

[18] Parsetree. http://rubyforge.org/projects/parsetree/.

[19] E. D. Sciascio, F. M. Donini, M. Mongiello, R. Totaro, and D. Castelluccia. Design verification of web applications using symbolic model checking. In Proc. 5th Int. Conf. Web Engineering (ICWE), pages 69-74, 2005.

[20] P. D. Stotts, R. Furuta, and C. R. Cabarrus. Hyperdocuments as automata: Verification of trace-based browsing properties by model checking. ACM Trans. Inf. Syst., 16(1):1-30, 1998.

[21] Tracks. http://getontracks.org/.

[22] L. Wang, G. Dobbie, J. Sun, and L. Groves. Validating ORA-SS data models using Alloy. In 17th Australian Software Engineering Conference (ASWEC), pages 231-242, 2006.

[23] S. Yuen, K. Kato, D. Kato, , and K. Agusa. Web automata: A behavioral model of web applications based on the MVC model. Information and Media Technologies, 1(1):66-79, 2006. 